\title{
External application of liver compresses to reduce fatigue in patients with metastatic cancer undergoing radiation therapy, a randomized clinical trial
}

Pirus Ghadjar ${ }^{*}$, Wiebke Stritter ${ }^{2}$, Irina von Mackensen², Felix Mehrhof ${ }^{1}$, Clara Foucré ${ }^{2}$, Vincent H. Ehrhardt ${ }^{\text {, }}$ Marcus Beck ${ }^{1}$, Pimrapat Gebert ${ }^{3}$, Goda Kalinauskaite ${ }^{1}$, Jacqueline S. Luchte ${ }^{2}$, Carmen Stromberger ${ }^{1}$, Volker Budach', Angelika Eggert ${ }^{2}$ and Georg Seifert ${ }^{2,4}$

\begin{abstract}
Background: Liver compresses are frequently used in integrative medicine as supportive therapy during cancer treatment in order to reduce fatigue. We performed a pilot study to test whether the external application of yarrow liver compresses impacts fatigue in patients with metastatic cancer undergoing radiation therapy.

Methods: A randomized prospective pilot trial was performed including patients with brain metastasis or bone metastasis of solid tumors. Patients underwent either palliative radiation therapy (RT) of the metastatic lesions (control group) over two weeks or the same RT with additional external application of yarrow liver compresses once daily during RT. The primary objective was improvement on the general fatigue subscale of the multidimensional fatigue inventory (MFI-20) at the end of treatment, where a mean difference of two points is considered clinically relevant. Secondary objectives included psychological distress, quality of life and qualitative analysis with self-established visual analogue scales (VAS). Mean differences in general fatigue at the end of treatment compared to baseline were analyzed using the ANCOVA test.

Results: From 09/2017 to 08/2019 a total of 39 patients were randomized. Due to drop outs 24 patients (12 per group) were available for analysis. Patients in the intervention group received a mean number of 10.5 (range, 7-14) applications of yarrow liver compresses. The mean improvement at the end of therapy on the general fatigue subscale of the MFI-20 was 2 points in favor of the intervention group $(p=0.13)$, and all other MFI-20 subscales showed at least a trend towards improvement in favor of the intervention group. Likewise, psychological distress and VAS data was improved, the latter reaching statistical significance for the symptoms fatigue, tension and lack of drive. Major toxicities were not observed.
\end{abstract}

Conclusions: External application of liver compresses appears to reduce fatigue within a clinical relevant range in patients with metastatic cancer undergoing radiation therapy.

\footnotetext{
*Correspondence: pirus.ghadjar@charite.de

1 Department of Radiation Oncology, Charité Universitätsmedizin Berlin, corporate member of Freie Universität Berlin, Humboldt-Universität zu Berlin and Berlin Institute of Health, Augustenburger Platz 1, 13353 Berlin, Germany

Full list of author information is available at the end of the article
}



(c) The Author(s) 2021. Open Access This article is licensed under a Creative Commons Attribution 4.0 International License, which permits use, sharing, adaptation, distribution and reproduction in any medium or format, as long as you give appropriate credit to the original author(s) and the source, provide a link to the Creative Commons licence, and indicate if changes were made. The images or other third party material in this article are included in the article's Creative Commons licence, unless indicated otherwise in a credit line to the material. If material is not included in the article's Creative Commons licence and your intended use is not permitted by statutory regulation or exceeds the permitted use, you will need to obtain permission directly from the copyright holder. To view a copy of this licence, visit http://creativecommons.org/licenses/by/4.0/. The Creative Commons Public Domain Dedication waiver (http://creativeco mmons.org/publicdomain/zero/1.0/) applies to the data made available in this article, unless otherwise stated in a credit line to the data. 
Trial registration: ISRCTN, ICTRP DRKS00012999

Keywords: External application, Compress, Cancer, Metastasis, Radiation therapy, Fatigue, Integrative medicine

\section{Background}

Fatigue has been described to be present in $\geq 30 \%$ of cancer patients and adversely impacts upon quality of life (QoL) [1]. The causation of fatigue is complex and often multifactorial, and treatment exists only for those patients in whom the cause can be targeted therapeutically, e.g. in patients with anemia causing fatigue who can receive transfusions. When no causal treatment for fatigue is available, across-the-board approaches such as increase of physical activity, treatment of possible sleep disorders and adherence to a balanced diet are advised, commonly only with modest success.

Randomized trials have demonstrated fatigue reduction through various interventions such as yoga [2], eurythmy therapy [3] and sport [4], but these interventions are not regularly performed.

Patients who undergo palliative radiation therapy (RT) for brain or bone metastases are commonly frail patients suffering symptoms caused by the disease and the treatment and fatigue has been shown to increase during and three months after RT [5, 6].

Yarrow (Achillea millefolium) is a medical plant which has been described as an analeptic and is perceived as warming, anticonvulsant and tonic according to largely unconfirmed anthroposophic medicine theories originating from Germany [7-9]. However, clinical trials are lacking on this topic [10]. Nevertheless, yarrow liver compresses are commonly used in integrative medicine as supportive therapy during cancer treatment due to its hepatoprotective qualities, amongst others to reduce fatigue. While aspects of palliative care already strongly impact on daily practice in radiation oncology [11] skepticism exists towards integrative approaches and its effects [12].

We conducted a prospective pilot trial to explore the potential fatigue improving effect of an external application of yarrow liver compresses in metastatic cancer patients undergoing RT.

\section{Patients and methods Patient selection}

This study was approved by the Ethical Committee of the Charité Universitätsmedizin Berlin, Germany (Reference number EA1/078/17) and the pilot trial was registered in the Cochrane Central Register of Controlled Trials (DRKS00012999).

For logistical reasons the decision was made to conduct this trial among patients with metastatic cancer undergoing radiation therapy being on an inpatient basis within the Department of Radiation Oncology, Charité Universitätsmedizin Berlin.

The criteria for patient eligibility were age $\geq 18$ years, with at least minor fatigue according to the general fatigue subscale of the multidimensional fatigue inventory (MFI-20) [13], estimated life expectancy $>3$ months, Karnofsky performance score $\geq 60 \%$, indication for inpatient palliative whole brain radiation therapy (WBRT) or palliative analgesic RT of bone metastasis. Patients were required to be inpatients due to the foreseen logistic hurdles in preparing and applying liver compresses in an outpatient setting. Patient written consent was mandatory. Patients were ineligible when severe psychiatric disorders or allergies prohibited participation and in cases with medical conditions potentially causing fatigue such as severe hypothyroidism, sleep apnea, insomnia, anemia with hemoglobin levels $<8 \mathrm{~g} / \mathrm{dL}$, cachexia with a body mass index $<18.5$, chronic kidney failure or acute depression. Patients with liver metastasis were also ineligible because in this case warming compresses were contraindicated, as were patients participating in other clinical trials. Patients receiving both WBRT and bone RT were also ineligible.

\section{Treatment}

Patients in both groups were treated by external beam RT. All patients underwent a dedicated planning computed tomography scan. For WBRT patients received 10 fractions of 3 Gy over 2 weeks using a three-dimensional conformal RT (3D-CRT) approach, wearing a thermoplastic mask for immobilization. Patients with bone metastasis received either 10 fractions of $3 \mathrm{~Gy}$ over 2 weeks or a slightly intensified regimen in the case of soft tissue tumor mass with up to 12-13 fractions of 3 Gy using 3D-CRT or an intensity modulated radiation therapy (IMRT) or volumetric modulated arc treatment (VMAT) approach. For WBRT the brain was defined as gross tumor volume (GTV) surrounded by a $5 \mathrm{~mm}$ margin to establish the planning target volume (PTV). For the treatment of bone metastasis, the involved bones/ bone regions were defined as GTV and a 5-10 $\mathrm{mm}$ margin added to establish the PTV. RT was performed using a 6-MV linear accelerator with multileaf collimators (Varian, USA).

During the inpatient course of treatment patients in both groups were offered palliative care in the form of visits from a dedicated multi-professional palliative care 
team of physicians, nurses, psycho-oncologists, physiotherapists and social workers, depending on the requirements of the respective patient.

External application of yarrow liver compresses was performed once daily over the course of two weeks of RT, the minimum number of applications was set to seven. Applications were preferably performed after lunch or in the evening. The liver compresses were prepared and applied by trained members of our scientific team in cooperation with the staff on the ward. For preparation of the compresses a dedicated room on the in-patient ward outside the patient room was used. One tablespoon of yarrow tea with cut blossoms, stems and leaves of common yarrow was infused with $500 \mathrm{ml}$ boiling water and brewed for $10 \mathrm{~min}$. The infusion was then strained and cooled for $5 \mathrm{~min}$ at room temperature to reach the required application temperature of $60^{\circ} \mathrm{C}$. A muslin cloth (made of cotton) was folded to the size of $20 \times 30 \mathrm{~cm}$, rolled up firmly (into a small roll) and placed on a rough cotton cloth (such as a tea towel). Both together were rolled up tightly, twisting the rough cotton cloth so that the two ends formed handles. This roll was then placed in a bowl. The necessary materials where brought to the patient's room where the patient was then comfortably positioned on the bed lying on an outer wrap. The wound-up roll in the bowl was then doused with the $60{ }^{\circ} \mathrm{C}$ hot yarrow infusion and wrung out firmly, wearing gloves to protect the hands from the heat. The muslin cloth was removed, carefully placed on the patient's right upper abdomen and lower costal arch. In case the patient experienced the muslin cotton as too hot, it was fanned to make the heat bearable. It was then covered with a cotton compress cloth which had been previously folded to the size of $20 \times 30 \mathrm{~cm}$. The outer wrap underneath the patient's back was then folded tightly over the compress from both sides. A hot-water bottle was placed on top and could be removed at the patient's convenience. The patient was then covered with a blanket and allowed to rest for 20-25 min. Care was taken to leave the patient alone in the room without external disturbance during the rest period. After $20-25$ min the moist inner muslin cloth was removed while the compress cloth and outer wrap stayed in place, including the hot-water bottle and the patient left to rest for another $30 \mathrm{~min}$ (Fig. 1).
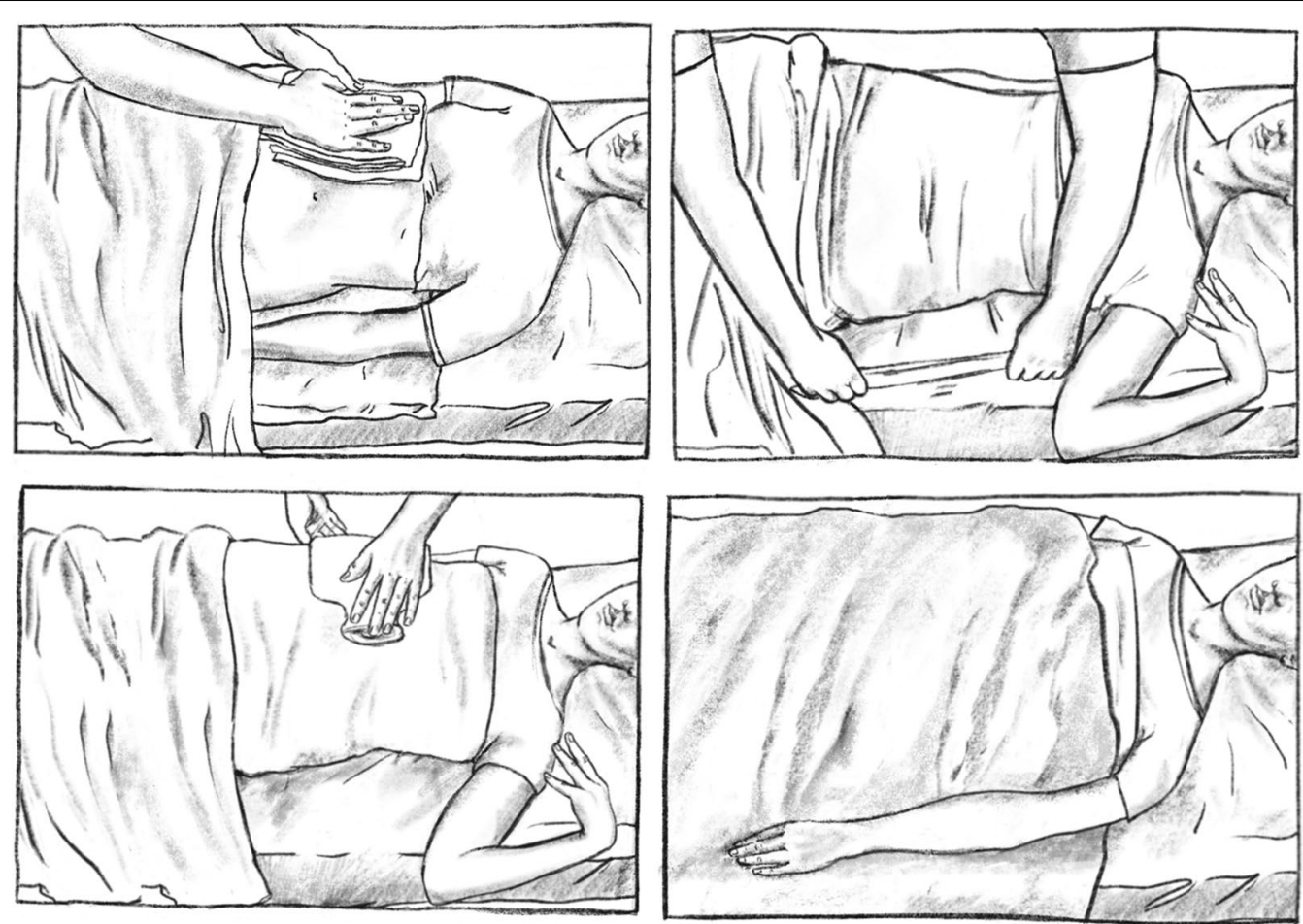

Fig. 1 Step-wise external application of a yarrow liver compress with careful placement of the inner muslin cloth, which had been doused with a $60^{\circ} \mathrm{C}$ hot yarrow infusion and then firmly wrung out, and the cotton compress cloth (on top of the inner cloth) on the right upper abdomen and lower costal arch of the comfortably positioned patient (top left); the inner and compress cloth were then covered with an outer wrap underneath the patient's back which was folded over from both sides (top right); a hot-water bottle was placed on top of the compress (bottom left); the patient was then covered with a blanket and allowed to rest for a total duration of 50-55 min (bottom right) 


\section{Measurements and endpoints}

Fatigue was assessed at baseline, after one week and at the end of the treatment according to the German version of the MFI-20 [13]. The MFI-20 is a 20-item psychometric inventory designed to evaluate five dimensions of fatigue: general fatigue, physical fatigue, reduced motivation, reduced activity, and mental fatigue [14]. The primary trial endpoint was the general fatigue subscale of the MFI-20 at the end of treatment. A mean difference of two points compared to baseline was considered clinically relevant [15].

Secondary endpoints included psychological distress as measured according to the German version of the distress thermometer of the National Comprehensive Cancer Network (NCCN) [16], patient's quality of life was assessed according to the European Organization for Research and Treatment of Cancer (EORTC) QLQ-C30 questionnaire [17]. Moreover, a qualitative analysis of patients of the intervention group was performed using questions regarding their condition on self-established visual analogue scales (VAS). The VAS covered tension, restlessness, fatigue, lack of drive, exhaustion, experience of warmth (cold hands/feet) and pain. All questionnaires were administered at baseline, after one week of treatment and at the end of treatment. As a sub-project, physiological data was assessed using heart rate variability and body temperature, which will be reported separately. Liver compress-related symptoms were assessed after each application and the time of onset and resolution as well as the intensity (mild vs. intermediate vs. severe) was noted.

\section{Statistical analyses}

The trial was designed as a randomized, non-blinded prospective clinical pilot trial. A pilot phase was planned with subsequent sample size recalculation. Block randomization was performed with stratification according to the RT site brain vs. bone.

The power calculation for the pilot phase was based on a 3-point reduction on the general fatigue subscale of the MFI-20 in the intervention group vs. control group at the end of treatment compared to baseline. With a common standard deviation of 3 points, alpha 0.05 and a power of $80 \%$ an initial sample size of 34 patients (17 per group) was required for an unpaired t-test. Accounting for $10 \%$ drop-outs 38 patients should initially be included. Due to the unblinded non-confirmatory design were $\mathrm{p}$-values are regarded as exploratory, no alpha-adjustment was planned. The sample size calculation was performed using $G^{*}$ Power Version 3.1.3.

Analysis of the pilot trial was performed using analysis of covariance (ANCOVA) with end and baseline differences on the general fatigue subscale as dependent and group variables, RT site brain vs. bone (strata) as independent variables and baseline general fatigue as covariate variable. A $p$ value $<0.05$ will be regarded as significant. $P$-values were not corrected for multiple testing. The effect size partial eta squared $\left(\eta^{2}\right)$ was presented to show the difference between groups as well as omega squared $\left(\omega^{2}\right)$ for correcting biased from a small sample size [18].

After the pilot trial had experienced severe accrual problems, due to many patients being ineligible and several other patients withdrawing from the trial after inclusion (Fig. 1), the pilot trial was halted in September 2019. Given the mean difference of two points on the general fatigue subscale (considered clinically relevant) in favor of the intervention group already found, the trial team decided to stop the pilot trial and moved ahead to analyze the results. All statistical tests were performed using Stata IC15 (StataCorp, 2017, College Station, TX, USA).

\section{Results}

Between September 2017 and August 2019 a total of 79 preselected patients were screened, of whom 39 were randomized, and after exclusion of drop outs a total of 24 patients (12 per group) were available for analysis (Fig. 2). The baseline patient characteristics are summarized in Table 1, there were no significant differences between the two pilot trial groups. Patients in the intervention group received a mean number of 10.5 applications of yarrow liver compresses (range 7-14).

For the primary endpoint, the general fatigue subscale of the MFI-20, we observed a mean improvement over baseline at the end of treatment of 2 points in favor of the intervention group $\left(p=0.13\right.$, effect size $\left.\left(\omega^{2}\right)=0.062\right)$ (Table 2 and Fig. 3). All other MFI-20 subscales tended in the same direction towards improvement of fatigue in patients of the intervention group. For the MFI-20 subscale reduced motivation the mean improvement at the end of therapy was 2.1 points the difference being statistically significant between the pilot trial groups $(\mathrm{p}=0.035$, effect size $\left(\omega^{2}\right)=0.158$ ) (Fig. 3). Mean changes from baseline (t0) at one week (t1) and at the end of treatment ( $\mathrm{t} 2)$ for all MFI-20 subscales are provided in the Additional file 1: Fig. 4.

Differences in psychological distress also tended to favour the intervention group (Table 2). Moreover, the VAS results showing significant improvement in the symptoms fatigue, tension and lack of drive in patients from the intervention group when compared to the control group also suggested a positive effect of the intervention (Table 2). There was no difference in the global health status/quality of life between the two groups (Table 2). 


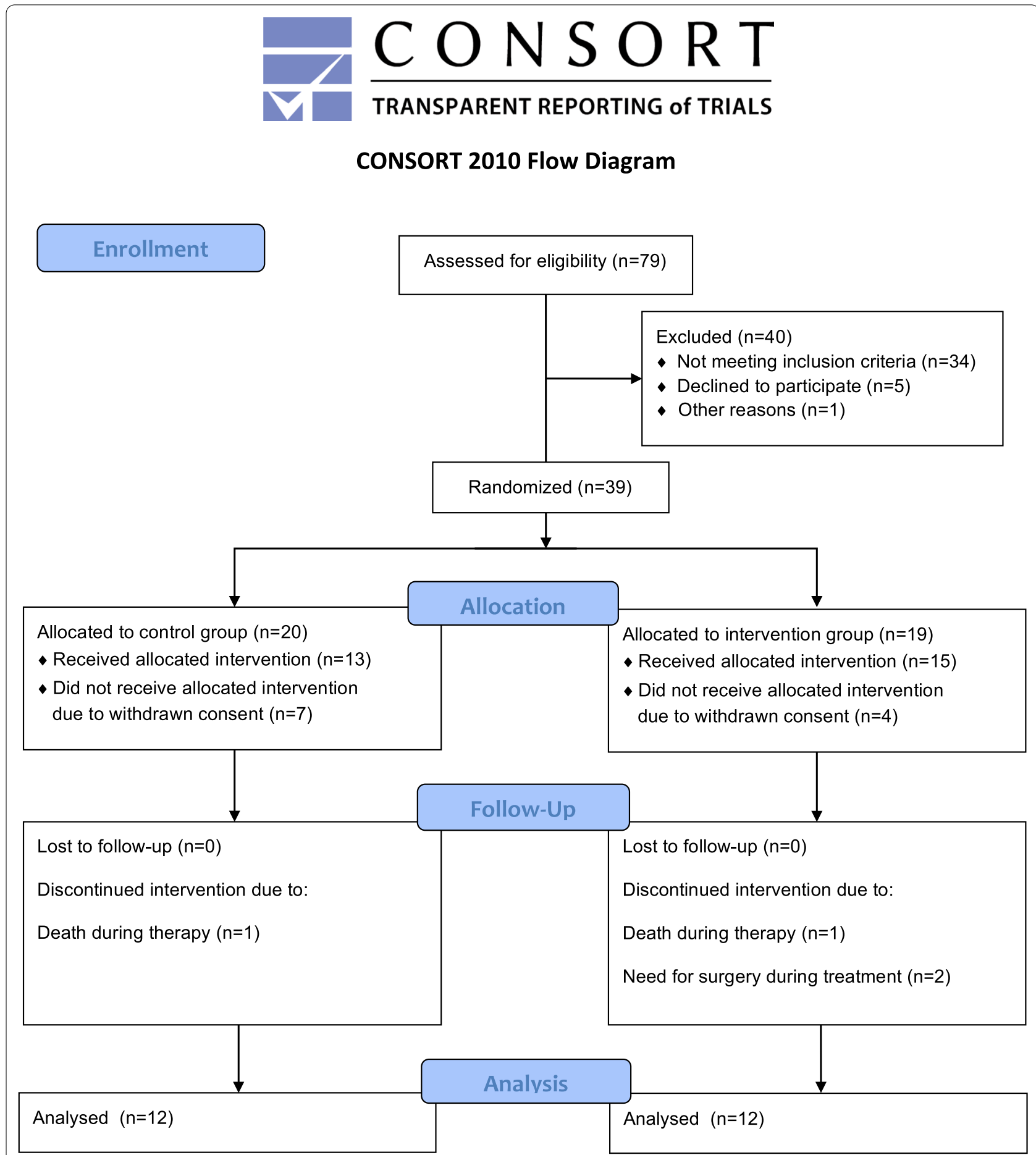

Fig. 2 Consort diagram of the trial

There were no significant differences between patients treated with WBRT or bone metastasis with respect to reduction of fatigue.
In five patients from the intervention group (42\%) local redness of the skin was observed at least once after the application of the compress which was not 
Table 1 Patient characteristics

\begin{tabular}{|c|c|c|c|}
\hline & $\begin{array}{l}\text { Radiotherapy alone } \\
(\mathrm{n}=12)\end{array}$ & $\begin{array}{l}\text { Radiotherapy and Yarrow compress } \\
(n=12)\end{array}$ & $\begin{array}{l}\text { Total } \\
{[24]}\end{array}$ \\
\hline \multicolumn{4}{|l|}{ Gender } \\
\hline Male & $3(25 \%)$ & $3(25 \%)$ & $6(25 \%)$ \\
\hline Female & $9(50 \%)$ & $9(50 \%)$ & $18(75 \%)$ \\
\hline \multicolumn{4}{|l|}{ Age (years) } \\
\hline Median (min.-max.) & $60.5(49-83)$ & $56.5(35-68)$ & $58.5(34-83)$ \\
\hline \multicolumn{4}{|l|}{ Karnofsky-Index } \\
\hline $80-100$ & $4(33 \%)$ & $3(25 \%)$ & $7(30 \%)$ \\
\hline 70 & $5(42 \%)$ & $4(33 \%)$ & $9(37 \%)$ \\
\hline 60 & $3(25 \%)$ & $5(42 \%)$ & $8(33 \%)$ \\
\hline \multicolumn{4}{|l|}{ Site of RT } \\
\hline Bone & $6(50 \%)$ & $7(58 \%)$ & $13(54 \%)$ \\
\hline Brain & $6(50 \%)$ & $5(42 \%)$ & $11(46 \%)$ \\
\hline \multicolumn{4}{|l|}{ Baseline Fatigue* } \\
\hline Median (min.-max.) & $14(10-20)$ & $16(10-20)$ & $14.5(10-20)$ \\
\hline
\end{tabular}

* Based on the general fatigue subscale of the multidimensional fatigue inventory (MFI-20)

perceived as uncomfortable by the patient and completely returned to normal without intervention. One patient developed mild nausea and in another patient a single episode of temporary localized crampy pain in the liver region was observed after application of the compress. Treatments were continued in both patients

Table 2 Comparison of fatigue and other patient reported outcomes between the trial arms

\begin{tabular}{|c|c|c|c|c|c|c|c|c|}
\hline & Intervention & Control & $\begin{array}{l}\text { Mean } \\
\text { difference* } \\
(I-C)\end{array}$ & $95 \% \mathrm{Cl}$ & p-value & $\begin{array}{l}\text { Effect size } \\
\left(\text { Partial } \eta^{2}\right)\end{array}$ & Effect size $\left(\omega^{2}\right)$ & \\
\hline & Mean (SD) & Mean (SD) & & Lower & Upper & & & \\
\hline MFI subscales (T0-T2) & $(n=12)$ & $(n=12)$ & & & & & & \\
\hline General Fatigue & $1.08(2.57)$ & $-0.50(3.75)$ & 1.98 & -0.65 & 4.60 & 0.132 & 0.110 & 0.062 \\
\hline Physical Fatigue & $0.83(4.17)$ & $0.42(4.74)$ & 1.44 & -1.60 & 4.48 & 0.336 & 0.046 & -0.001 \\
\hline Reduced Activity & $1.75(2.96)$ & $0.58(4.68)$ & 1.69 & -1.08 & 4.45 & 0.218 & 0.075 & 0.027 \\
\hline Reduced Motivation & $0.08(3.06)$ & $-2.58(2.43)$ & 2.14 & 0.17 & 4.12 & 0.035 & 0.204 & 0.158 \\
\hline Mental Fatigue & $1.25(3.31)$ & $0.00(4.09)$ & 1.03 & -0.87 & 2.92 & 0.273 & 0.060 & 0.012 \\
\hline $\begin{array}{l}\text { Psychological Distress } \\
\text { (T0-T2) }\end{array}$ & $(n=12)$ & $(n=12)$ & & & & & & \\
\hline Distress & $0.82(2.27)$ & $-0.15(2.24)$ & 1.08 & -0.70 & 2.87 & 0.218 & 0.088 & 0.032 \\
\hline VAS (T2-T0) & $(n=11)$ & $(n=10)$ & & & & & & \\
\hline VAS1 Tension & $0.64(1.29)$ & $-0.80(1.42)$ & 1.14 & 0.04 & 2.25 & 0.044 & 0.219 & 0.165 \\
\hline VAS2 Restlessness & $0.55(1.04)$ & $-0.35(1.13)$ & 0.77 & -0.10 & 1.64 & 0.080 & 0.169 & 0.115 \\
\hline VAS3 Fatigue & $0.64(1.38)$ & $-0.60(0.81)$ & 1.12 & 0.25 & 1.98 & 0.015 & 0.301 & 0.250 \\
\hline VAS4 Lack of drive & $0.36(1.63)$ & $-0.45(1.26)$ & 0.97 & 0.12 & 1.82 & 0.028 & 0.253 & 0.201 \\
\hline VAS5 Exhaustion & $0.41(1.18)$ & $-0.20(0.79)$ & 0.76 & -0.05 & 1.57 & 0.063 & 0.189 & 0.135 \\
\hline VAS6 Cold hands/feet & $0.32(1.71)$ & $0.15(0.91)$ & 0.25 & -0.77 & 1.28 & 0.608 & 0.016 & -0.040 \\
\hline VAS7 Pain & $0.95(1.67)$ & $-0.10(1.51)$ & 0.75 & -0.48 & 1.99 & 0.215 & 0.089 & 0.034 \\
\hline EORTC QLQ-C30 (T2-T0) & $(n=12)$ & $(n=12)$ & & & & & & \\
\hline Global health status/QoL & $6.94(25.08)$ & $5.56(12.97)$ & 2.66 & -11.71 & 17.02 & 0.703 & 0.007 & -0.040 \\
\hline
\end{tabular}

$\mathrm{MFI}=$ multidimensional fatigue inventory; $\mathrm{SD}=$ standard deviation; $\mathrm{I}-\mathrm{C}=$ Intervention group-Control group; $\mathrm{Cl}=$ confidence interval; $\mathrm{\eta}^{2}=\mathrm{Partial}$ eta squared; $\omega^{2}=$ Omega squared; VAS = visual analogue scale; EORTC = European Organization for Research and Treatment of Cancer; QLQ = quality of life questionnaire; $\mathrm{QoL}=$ quality of life; ${ }^{*}$ Mean difference and $95 \% \mathrm{Cl}$ were calculated using ANCOVA with adjustment for baseline and RT sites (brain vs. bone) 


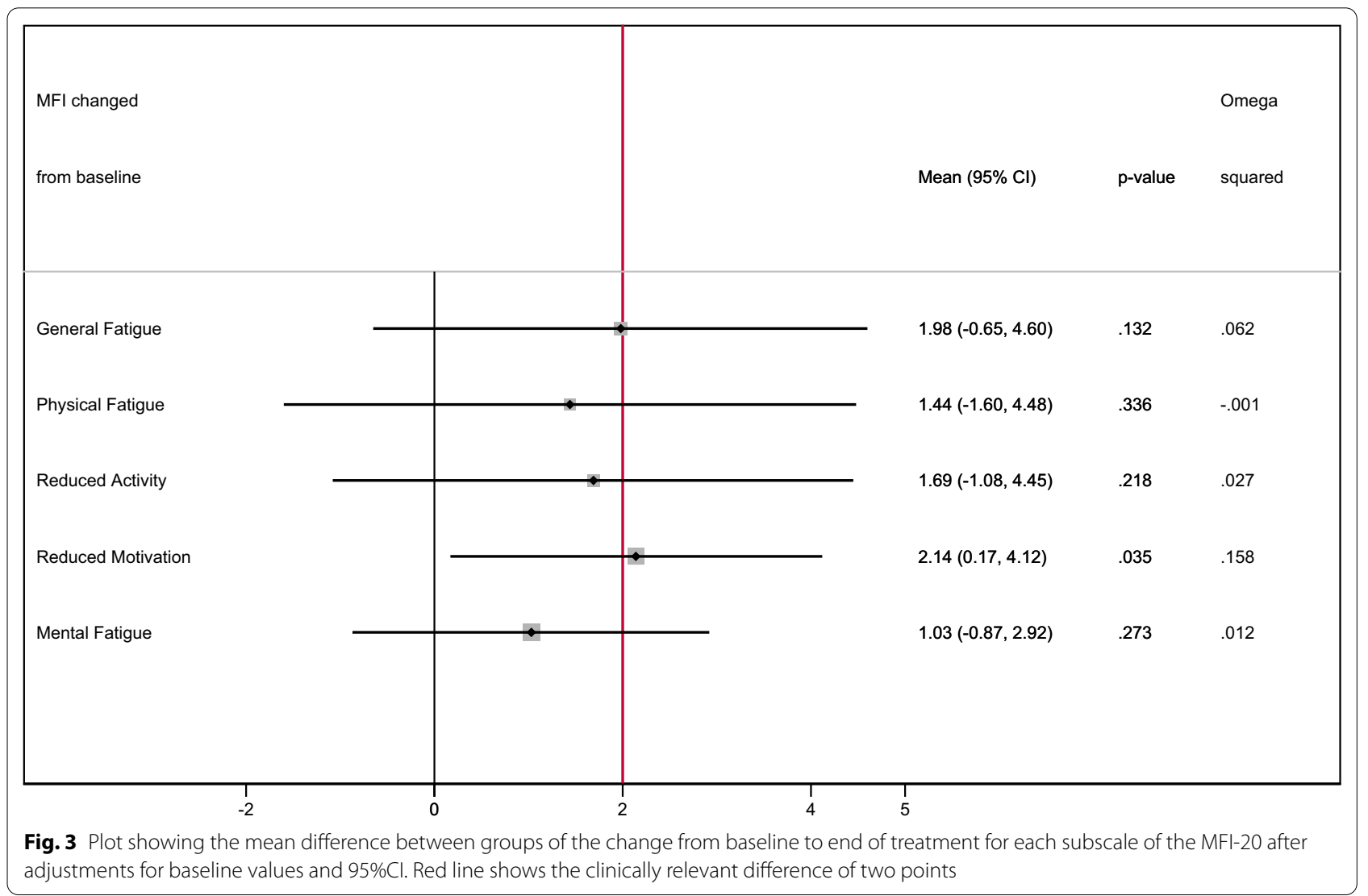

and neither of the two symptoms occurred again. Major compress-related toxicities were not observed.

\section{Discussion}

In this pilot trial we observed a clinically relevant reduction of fatigue after external application of yarrow liver compresses in patients with metastatic cancer undergoing RT. This is significant given the small cohorts available for analysis. All subscales of the MFI-20 tended in the same direction and other measures such as the psychological distress and VAS data were also supportive of the assumption that there is a true effect. In addition, it appears that yarrow liver compresses were tolerated well by patients with no major toxicity observed.

Patients undergoing WBRT for brain metastasis have a life expectancy of only 3-6 months and commonly experience intracranial recurrence and or recurrence of the extracranial disease [19]. Likewise, pain progression at the treated site and/or development of new bone metastasis are relatively common in patients with bone metastasis $[20,21]$. As these RT treatments, subsequent salvage $\mathrm{RT}$ treatments as well as additional systemic treatments can cause fatigue, and as patient reported symptoms at baseline were shown to predict survival [22], the identification of fatigue reducing well tolerated and cost effective strategies is very important. The time required for preparation of a yarrow liver compress is estimated to be 5-10 min without significant material expenses. In this respect, it is a simple method that can be applied quickly, and which certainly has a calming effect on patients and could thus save costs and time overall. Since other studies point to the anti-inflammatory and spasmolytic effect of yarrow [23-25], these patients might profit from this simple intervention on multiple levels.

Because of problems with patient recruitment the pilot trial was prolonged for a total duration of 2 years but its continuation was subsequently impossible. On account of the pilot nature of this trial the decision was made to perform early data analysis. A high number of screened patients could not be included in the pilot trial as they did not meet the inclusion criteria of at least minor fatigue. This needs to be accounted for in a subsequent trial in another group of cancer patients. However, it is unknown whether yarrow liver compresses might even be more effective in a patient population with a higher degree of baseline fatigue.

It is also not known, relating to the non-blinded character of our pilot trial, whether the observed fatigue reduction was caused by the yarrow itself, or the application of the liver compresses, due to the attention towards 
the patient during the whole procedure, due to a placebo effect or due to a combination of the above. Exploring the exact mechanism of action is somewhat unimportant from a patient perspective as patients do appear to benefit from the compress. However, from a scientific point of view it might be worth performing a placebo-controlled trial e.g. liver compress with and without yarrow, to further expand the knowledge on external applications.

\section{Conclusions}

External application of liver compresses appears to reduce fatigue in patients with metastatic cancer undergoing palliative radiation therapy.

\section{Abbreviations}

Cl: Confidence interval; EORTC: European Organization for Research and Treatment of Cancer; GTV: Gross tumor volume; I-C: Intervention group - Control group; IMRT: Intensity modulated radiation therapy; MFI: Multidimensional fatigue inventory; NCCN: National Comprehensive Cancer Network; PTV: Planning target volume; RT: Radiation therapy; SD: Standard deviation; VAS: Visual analogue scale; VMAT: Volumetric modulated arc treatment; WBRT: Whole brain radiation therapy; QLQ: Quality of life questionnaire; QoL: Quality of life; 3D-CRT: Three-dimensional conformal RT.
\end{abstract}

\section{Supplementary Information}

The online version contains supplementary material available at https://doi. org/10.1186/s13014-021-01757-x.

Additional file 1. Figure 4: Line plot showing mean changes from baseline (t0) at one week (t1) and at end of treatment (t2) for all MFI-20 subscales after adjustments for different baseline values.

\section{Acknowledgements}

Not applicable

\section{Authors' contributions}

Pirus Ghadjar: conception, organization, data analysis, manuscript writing, manuscript editing and final approval. Wiebke Stritter: conception, organization, data analysis, manuscript editing and final approval. Irina von Mackensen: data analysis, performance of yarrow liver compresses, manuscript editing and final approval. Felix Mehrhof: enrollment of patients, manuscript editing and final approval. Clara Foucré: data analysis, performance of yarrow liver compresses, manuscript editing and final approval. Vincent $H$. Ehrhardt: enrollment of patients, manuscript editing and final approval. Marcus Beck: enrollment of patients, manuscript editing and final approval. Pimrapat Gebert: statistical analysis, manuscript editing and final approval. Goda Kalinauskaite: enrollment of patients, manuscript editing and final approval. Jaqueline S. Luchte: data analysis, performance of yarrow liver compresses, manuscript editing and final approval. Carmen Stromberger: enrollment of patients, manuscript editing and final approval. Volker Budach: organization, manuscript editing and final approval. Angelika Eggert: organization, manuscript editing and final approval. Georg Seifert: conception, organization, data analysis, manuscript editing and final approval. All authors read and approved the final manuscript.

\section{Funding}

Open Access funding enabled and organized by Projekt DEAL. The Christophorus foundation supported this work with a grant (Grant Number 277CST). Christophorus Stiftung, GLS Treuhand,Kernerplatz 2, 70182 Stuttgart. The Helixor foundation supported this work with a grant (Grant Number 57/16 and 45/17). Helixor Heilmittel GmbH, Fischermühle 1, 72348 Rosenfeld.

\section{Availability of data and materials}

The datasets used and/or analyzed during the current study are available from the corresponding author on reasonable request.

\section{Ethics approval}

This study was approved by the Ethics Committee of the Charité Universitätsmedizin Berlin, Germany.

\section{Consent for publication \\ Not applicable.}

\section{Competing interests}

The authors declare that they have no competing interests.

\section{Author details}

${ }^{1}$ Department of Radiation Oncology, Charité Universitätsmedizin Berlin, corporate member of Freie Universität Berlin, Humboldt-Universität zu Berlin and Berlin Institute of Health, Augustenburger Platz 1, 13353 Berlin, Germany. 2 Division of Oncology and Hematology, Department of Pediatrics, Charité Universitätsmedizin Berlin, corporate member of Freie Universität Berlin, Humboldt-Universität zu Berlin and Berlin Institute of Health, Berlin, Germany. ${ }^{3}$ Institute of Biometry and Clinical Epidemiology, Charité Universitätsmedizin Berlin, corporate member of Freie Universität Berlin, Humboldt-Universität zu Berlin and Berlin Institute of Health, 13353 Berlin, Germany. ${ }^{4}$ Faculty of Medicine, Department of Paediatrics, University of São Paulo, São Paulo, Brazil.

Received: 12 October 2020 Accepted: 28 January 2021

Published online: 19 April 2021

\section{References}

1. Neefjes EC, van der Vorst MJ, Blauwhoff-Buskermolen S, Verheul HM. Aiming for a better understanding and management of cancer-related fatigue. Oncologist. 2013;18:1135-43.

2. Bower JE, Garet D, Sternlieb B, Ganz PA, Irwin MR, Olmstead R, Greendale G. Yoga for persistent fatigue in breast cancer survivors: a randomized controlled trial. Cancer. 2012;118:3766-75.

3. Kröz M, Reif M, Glinz A, Berger B, Nikolaou A, Zerm R, Brinkhaus B, Girke $M$, Büssing A, Gutenbrunner C; CRF-2 study group. Impact of a combined multimodal-aerobic and multimodal intervention compared to standard aerobic treatment in breast cancer survivors with chronic cancer-related fatigue - results of a three-armed pragmatic trial in a comprehensive cohort design. BMC Cancer. 2017;17:166

4. Dimeo F, Schwartz S, Wesel N, Voigt A, Thiel E. Effects of an endurance and resistance exercise program on persistent cancer-related fatigue after treatment. Ann Oncol. 2008;19:1495-9.

5. Strauss B, Brix C, Fischer S, Leppert K, Fuller J, Roehrig B, et al. The influence of resilience on fatigue in cancer patients undergoing radiation therapy (RT). J Cancer Res Clin Oncol. 2007;133:511-8.

6. Steinmann D, Vordermark D, Gerstenberg W, Aschoff R, Gharbi N, Müller A, Schäfer C, Theodorou M, Wypior HJ, Geinitz H; Quality of Life Working Group of the German Radiation Oncology Society (DEGRO). Quality of life in patients with limited (1-3) brain metastases undergoing stereotactic or whole brain radiotherapy: A prospective study of the DEGRO QoL working group. Strahlenther Onkol. 2020;196:48-57.

7. Akram M. Minireview on Achillea millefolium Linn. J Membr Biol. 2013;246:661-3.

8. Deckers B. Von der Sinnfrage der Krebskrankheit zur Pflege der Sinne. In R. Heine (Ed.), Anthroposophische Pflegepraxis 2015;pp. 411-453. Berlin: Salumed.

9. Fingado M. Therapeutische Wickel und Kompressen. Dornach: 2012 Natura Verlag.

10. Mühlenpfordt I, Stritter W, Bertram M, Ben-Arye E, Seifert G. The power of touch: external applications from whole medical systems in the care of cancer patients (literature review). Support Care Cancer. 2020;28:461-71.

11. Fels J, Pigorsch S, Vorwerk H, Engenhart-Cabillic R, van Oorschot B. Palliative care in everyday practice of radiation oncologists : Results from a web-based survey among medical members of the German Society for Radiation Oncology (DEGRO). Strahlenther Onkol. 2019;195:659-67. 
12. Kessel KA, Klein E, Hack CC, Combs SE. Complementary medicine in radiation oncology: German health care professionals' current qualifications and therapeutic methods. Strahlenther Onkol. 2018;194:904-10.

13. Schwarz R, Krauss O, Hinz A. Fatigue in the general population. Onkologie. 2003;26:140-4.

14. Smets EM, Garssen B, Bonke B, De Haes JC. The Multidimensional Fatigue Inventory (MFI) psychometric qualities of an instrument to assess fatigue. J Psychosom Res. 1995;39:315-25.

15. Purcell A, Fleming J, Bennett S, Burmeister B, Haines T. Determining the minimal clinically important difference criteria for the Multidimensional Fatiguelnventory in a radiotherapy population. Support Care Cancer. 2010;18:307-15.

16. Mehnert A, Müller D, Lehmann C, Koch U. Die deutsche Version des NCCN Distress Thermometers - Empirische Prüfung eines ScreeningInstruments zur Erfassung psychosozialer Belastung bei Krebspatienten. Zeitschrift für Psychiatrie, Psychologie und Psychotherapie. 2006;54:213-23.

17. Aaronson NK, Ahmedzai S, Bergman B, Bullinger M, Cull A, et al. The European Organization for Research and Treatment of Cancer QLQ-C30: a quality-of-life instrument for use in international clinical trials in oncology. J Natl Cancer Inst. 1993;85:365-76.

18. Lakens D. Calculating and reporting effect sizes to facilitate cumulative science: a practical primer for t-tests and ANOVAs. Front Psychol. 2013;4:863.

19. Tsao MN, Xu W, Wong RK, Lloyd N, Laperriere N, Sahgal A, et al. Whole brain radiotherapy for the treatment of newly diagnosed multiple brain metastases. Cochrane Database Syst Rev. 2018;1:CD003869.
20. Chow E, Harris K, Fan G, Tsao M, Sze WM. Palliative radiotherapy trials for bone metastases: a systematic review. J Clin Oncol. 2007;25:1423-36.

21. Bostel T, Förster R, Schlampp I, Sprave T, Akbaba S, Wollschläger D, Debus J, Mayer A, Schmidberger H, Rief H, Nicolay NH. Stability and survival analysis of elderly patients with osteolytic spinal bone metastases after palliative radiotherapy : Results from a large multicenter cohort. Strahlenther Onkol. 2019;195:1074-85.

22. Nieder C, Kämpe TA, Pawinski A, Dalhaug A. Patient-reported symptoms before palliative radiotherapy predict survival differences. Strahlenther Onkol. 2018;194:533-8.

23. Miranzadeh S, Adib-Hajbaghery M, Soleymanpoor L, Ehsani M. Effect of adding the herb Achillea millefolium on mouthwash on chemotherapy induced oral mucositis in cancer patients: a double-blind randomized controlled trial. Eur J Oncol Nurs. 2015;19:207-13.

24. Jenabi E, Fereidoony B. Effect of Achillea Millefolium on relief of primary dysmenorrhea: a double-blind randomized clinical trial. J Pediatr Adolesc Gynecol. 2015;28:402-4.

25. Freysdottir J, Logadottir OT, Omarsdottir SS, Vikingsson A, Hardardottir I. A polysaccharide fraction from Achillea millefolium increases cytokine secretion and reduces activation of Akt, ERK and NF-kappaB in THP-1 monocytes. Carbohydr Polym. 2016;143:131-8.

\section{Publisher's Note}

Springer Nature remains neutral with regard to jurisdictional claims in published maps and institutional affiliations.
Ready to submit your research? Choose BMC and benefit from:

- fast, convenient online submission

- thorough peer review by experienced researchers in your field

- rapid publication on acceptance

- support for research data, including large and complex data types

- gold Open Access which fosters wider collaboration and increased citations

- maximum visibility for your research: over $100 \mathrm{M}$ website views per year

At BMC, research is always in progress.

Learn more biomedcentral.com/submissions 